\title{
Further properties of L-closed topological spaces
}

\author{
Eman Almuhur ${ }^{1}$, Manal Al-labadi ${ }^{2}$ and Sarah Khamis ${ }^{3}$ \\ ${ }^{1}$ Department of Basic Science and Humanities, Applied Science Private University, Amman, Jordan, \\ ${ }^{2}$ Department of Mathematics, University of Petra, Amman, Jordan \\ ${ }^{3}$ Department of Business Administration, Lusail University, Doha, Qatar
}

Received: 13 June 2021, Accepted: 16 June 2021

Published online: 4 July 2021.

\begin{abstract}
The main purpose of this research paper is to discuss properties of L-closed spaces in topology and propose further properties concerning this concept. In addition, we explored the relationships between L-closed spaces and other topological spaces.
\end{abstract}

Keywords: L-closed space, L-closed neighborhood, L-closed property.

\section{Introduction}

In 1983, Hdeib and Pareek [6] introduced a new topological space called L-closed space in which each Lindelöf subset of a topological space $(X, \tau)$ is closed. Lots of results concerning the new notion are obtained and they posed two queries, the first query is: "Does there exists a regular L-closed space which are not a P-spaces?"

The second one is: "In an L-closed space, every countable subset is closed, when is the inverse true?". In 1985, two mathematicians Henriksen and Woods [17] presented their answers to the queries within the Tychonoff spaces.

In this paper, we will denote a topological space $(\mathrm{X}, \tau)$ by $X, \mathbb{R}$ and $\mathbb{N}$ denote the set of all real and natural numbers respectively. Moreover, $\tau_{c o c}, \tau_{d i s}, \tau_{u}, \tau_{s}, \tau_{c o f}, \tau_{l}$ and $\tau_{r}$, will denote cocountable, discrete, usual, Sorgenfrey, cofinite, left ray, and right ray opologies. Finally, the closure and interior of a set in a space say $U$ will be denoted by $c l U$ and $i n t U$ respectively.

\section{Preliminaries}

Studying compact and Lindelöf topological spaces requires a deep understand of the nature of their covers, Fletcher and other topologists [5] displayed the definitions of open cover where a cover (indexed family) $C=\left\{U_{\alpha}: \alpha \in \Lambda\right\}$ of a non-empty space $X$ is a collection of subsets whose union is $X$.

Lots of topologists focused on the importance of covers in determining the nature and the type of a space, for instance, Fletcher [5], Hoyle, Patty and Kim [9] proved that the product of pairwise compact spaces is not a pairwise compact space.

Lindelöf spaces, introduced by Alexandroff and Urysohn in 1929, have a highly expressive role in topology, it generalizes compact spaces.

\footnotetext{
*Corresponding author e-mail: E_almuhur@asu.edu.jo, manal.allabadi@uop.edu.jo and skhamis@1u.edu.qa @2021 BISKA Bilisim Technology
} 
The most familiar concepts in topology are "Separation axioms" that define topological classes and distinguish disjoint sets and distinct points [2]. Such classes of spaces are given the names $T_{1}, T_{2}, T_{3}$, and $T_{4}$ as an abbreviation of a German word "Trennung" which means separation. After there, system of numbering was extended to include $T_{0}, T_{2 \hat{A}^{1 / 2}}, T_{3 \hat{A}}{ }^{1 / 2}($ or $\left.\mathrm{T}_{\pi}\right), \mathrm{T}_{5}$, and $\mathrm{T}_{6}[3]$.

\section{L-closed Spaces}

In a space $X$, if every countable subset is closed, consequently every countable subset of such space is discrete, and every compact subset is finite [6], consequently every subset of L-closed space $X$ is closed and every subspace of an L-closed space is also L-closed.

Each Housdorff space is L-closed space and if $\forall x$ in a space $X$ has an L-closed neighborhood say $W$ where $\exists W$ a Lindelöf subset of $X$ containing $x$. Now, a regular space $X$. In regular spaces in which $\forall x$ has an L-closed neighborhood, a space will be L-closed. Moreover, a regular L-closed space is normal [14]. Now, a regular space $X$ that can be represented as a countable union of subspaces and each of such subspaces has L-closeness property has itself L-closeness property.

In a hereditarily Lindelöf space $X, X$ is L-closed if and only if it is countable and discrete. The sum $\bigoplus_{\alpha \in \Lambda} X_{\alpha}$ where $X_{\alpha} \neq \phi$ for some $\alpha \in \Lambda$ has L-closeness property if and only if each space $X_{\alpha}$ has the L-closeness property and $\Lambda$ is a countable set. Furthermore, a subset $A \subset \bigoplus_{\alpha \in \Lambda} X_{\alpha} \mathrm{X}$ is L-closed $\Longleftrightarrow A \cap X_{\alpha}$ is L-closed in $X_{\alpha}, \alpha \in \Lambda$.

A space $\mathrm{X}$ is $\mathrm{L}_{4}$-space [8] if $\forall A$ a Lindelöf subset of $\mathrm{X}, \exists F$ a Lindelöf $\mathrm{F}_{\sigma}$-subset of $X \ni A \subseteq F \subseteq \bar{A}$. Typically, each L-closed space is $\mathrm{L}_{4}$-space and as a consequence we have that in each L-closed space, every Lindelöf $\mathrm{F}_{\sigma}$-subset is closed. Now, if every Lindelöf subset of $X$ is a $\mathrm{F}_{\sigma}$-subset, then $\mathrm{X}$ is $\mathrm{T}_{1}$ and each closed countable subset of $\mathrm{X}$ will be discrete.

A space $X$ is $\mathrm{L}_{1}$ [8] if every Lindelöf $\mathrm{F}_{\sigma}$-subset of $\mathrm{X}$ is closed. Depending on this definition, an L-closed space is $\mathrm{L}_{1}$ space. The property of being pairwise $\mathrm{L}_{1}$-space is hereditarily property with respect to $\mathrm{F}_{\sigma}$-subsets.

A space $X$ is $\mathrm{L}_{3}$ [8] if every Lindelöf subset of $\mathrm{X}$ is an $\mathrm{F}_{\sigma}$-set. From this definition, we conclude that the property of being a $\mathrm{L}_{3}$-space is a hereditarily property and that $X$ is $\mathrm{L}$-closed $\Longleftrightarrow$ it is $\mathrm{L}_{1}$ and $\mathrm{L}_{3}$.

\section{Product properties of L-closed spaces}

Proposition 1. Let $(X, \tau)$ and $(Y, \sigma)$ be two spaces such that $Y$ is an L-closed space. If $f:(X, \tau) \rightarrow(Y, \sigma)$ is a continuous onto function, then $(X, \tau)$ is an L-closed space.

Proof. If $\mathrm{f}:(X, \tau) \rightarrow(Y, \sigma)$ is a continuous onto function. Let $(Y, \sigma)$ be an L-closed space, let $F$ be a Lindelöf subset of $X$, then $\mathrm{f}(F)$ is Lindelöf since $\mathrm{f}$ is a continuous function.

But $(Y, \sigma)$ is an L-closed space, thus $\mathrm{f}(F)$ is a closed subset of $Y$, and $F=\mathrm{f}^{-1}(\mathrm{f}(F))$ is a closed subset of $X$ because $\mathrm{f}$ is a one to one function. Therefore, $(X, \tau)$ is an L-closed space. Now, any L-closed topological space is homeomorphic to itself.

Proposition 2. Let $(X, \tau)$ and $(Y, \sigma)$ be two spaces such that $(X, \tau)$ is Lindelöf, $(Y, \sigma)$ is L-closed, if $f:(X, \tau) \rightarrow(Y, \sigma)$ is an onto continuous function, then $f$ is homeomorphism. 
Proof. We claim that $\mathrm{f}$ is a closed function. Let $\tilde{\mathscr{U}}$ be a closed family in $X$ such that $C \in \tilde{\mathscr{U}}$, so $C$ is a closed proper subset of $X$. Since $X$ is Lindelöf, then $C$ is a Lindelöf subset of $X$, hence $\mathrm{f}(C)$ is Lindelöf because $\mathrm{f}$ is continuous [10]. But $(Y, \sigma)$ is an L-closed space, so $\mathrm{f}(C)$ is a closed subset of $\mathrm{Y}$.

Proposition 3. If a continuous function from a Lindelöf Hausdorff space to an L-closed space is closed, then every continuous onto function is homeomorphism.

Proposition 4. Being L-closed space is a topological property.

Proof. Let $(X, \tau)$ be an L-closed space and $(Y, \sigma)$ be any space, let $h:(X, \tau) \rightarrow(Y, \sigma)$ be homeomorphism. If $A$ is a Lindelöf subset of $X$, then $\mathrm{h}(A)$ is Lindelöf because $h$ is continuous. If $X$ is an L-closed space and $A$ is closed, then $\mathrm{h}(A)$ is closed because $\mathrm{h}$ is a closed function. Thus $Y$ is an L-closed space.

Proposition 5. Let $(X, \tau)$ and $(Y, \sigma)$ be two spaces such that $X$ is $L$-closed. If $f:(X, \tau) \rightarrow(Y, \sigma)$ is any function and $\{(x, f(x))$ : $x \in X\}$ is a Lindelöf subset of $X \times Y$, then $f$ is continuous.

Proof. Let $\pi_{x}$ and $\pi_{y}$ be two projection functions. If $\pi_{x}^{\prime}=\pi_{x_{\mid f}}$, $\pi_{x}^{\prime}$ and $\pi_{y}$ are continuous surjective functions. If $\tilde{\mathscr{A}}=\{(x, \mathrm{f}(x)): x \in \mathrm{X}\}$. Since $\tilde{\mathscr{A}}$ is Lindelöf, any closed subset of $\mathrm{f}$ is Lindelöf, so $\pi_{x}^{\prime}$ is a closed projection function, i.e if $A \subseteq \mathrm{f}$ is a closed subset, so $A$ is a Lindelöf subset of $X$ because $X$ is L-closed space. Now, $\mathrm{f}$ is defined on $X$, so $\pi_{x}^{\prime}$ is a onto function. Using this fact and the fact that $\pi_{x}^{\prime}$ is a closed projection function, we get that open set $v \subseteq f$ we have $\pi_{x}^{\prime}(v)$ is open in $X$.

Hence $\mathrm{f}=\pi_{y} \circ\left(\pi_{x}^{\prime}\right)^{-1}$ is p-continuous.

Proposition 6. If the topological spaces $(X, \tau)$ and $(Y, \sigma)$ are $L$-closed such that either $X$ or $Y$ is regular, then $X \times Y$ is an L-closed space.

Proof. If $(X, \tau)$ and $(Y, \sigma)$ are L-closed spaces and let $\mathrm{Y}$ be regular. If $F$ is a Lindelöf subset of $\mathrm{X} \times \mathrm{Y}$. Let $\left(x_{0}, y_{0}\right) \in c l(F)$ $F$, so $\left(x_{0}, y_{0}\right) \notin\left[\left(\left\{x_{0}\right\} \times \mathrm{Y}\right] \cap F\right.$ and $\left(\left\{x_{0}\right\} \times Y\right) \cap F$ is a closed subset of $X \times Y$ because $\mathrm{Y}$ is an L-closed space. $\mathrm{Y}$ is regular, so $\exists H$ an open subset containing $y_{0}$ such that $(\mathrm{X} \times c l H) \cap\left[\left(\left\{x_{0}\right\} \times \mathrm{Y}\right) \cap F\right]=\phi$, so the projection function $\pi_{x}((\mathrm{X} \times$ $\left.c l_{2} H\right) \cap\left(\left(\left\{x_{0}\right\} \times \mathrm{Y}\right) \cap F\right)$ is a closed subset of $\mathrm{X}$ because $\pi_{x}$ is continuous [2]. $\left.\mathrm{X}-\left[\pi_{x}\left(\mathrm{X} \times c l_{2} H\right) \cap F\right) \times(\mathrm{Y} \cap(\mathrm{X} \times \mathrm{H}))\right]$ is an open neighborhood of $\left(x_{0}, y_{0}\right)$ disjoint from $F$, hence a subset $F$ is closed in $\mathrm{X} \times \mathrm{Y}$.

Proposition 7. The product of finite number of p-regular pairwise L-closed spaces is pairwise L-closed.

Proof. Let $\left\{\left(\mathrm{X}_{k}, \tau^{k}\right): k=1,2, \ldots, \mathrm{n}\right\}$ be a family of finitely many regular L-closed spaces. Let $\mathrm{X}=\prod_{k \in \mathbb{N}} \mathrm{X}_{k}$. By the induction on $k$, for $k=2$ the result is given by 3.2.1. Suppose that this is true for $k=n$, we claim that it is true for $k=n+1$. $\left(\mathrm{X}_{1} \times\right.$ $\left.\mathrm{X}_{2} \times \ldots \times \mathrm{X}_{n}\right) \times \mathrm{X}_{n+1}$ is homeomorphic to $\mathrm{X}_{1} \times \mathrm{X}_{2} \times \ldots \times \mathrm{X}_{n} \times \mathrm{X}_{n+1}$ and $\left(\mathrm{X}_{1} \times \mathrm{X}_{2} \times \ldots \times \mathrm{X}_{n}\right) \times \mathrm{X}_{n+1}$ is L-closed space by the assumption and using the fact that the product of two regular L-closed spaces. Hence $\mathrm{X}$ is L-closed [9].

From the previous prepositions we conclude that the finite product of L-closed regular spaces is L-closed, however infinite product of L-closed regular spaces needs not to be L-closed [12] as the following example shows, for example, if we consider that $X=\{0,1\}$ is endowed with two topologies that are discrete, then $X$ is regular L-closed [5]. Nevertheless, the countable product $\mathrm{Y}=\prod_{\alpha \in \Lambda} \mathrm{X}_{\alpha}$ is compact and infinite. Thus, a space $\mathrm{Y}$ cannot be $\mathrm{L}$-closed.

Proposition 8. Suppose that for the two spaces $(X, \tau)$ which is an L-closed space and $(Y, \sigma)$ is a Lindelö space, so the projection function $\pi_{x}: X \times Y \rightarrow X$ is Lindelöf.

Proof. Consider a Lindelöf subset $F$ of $\mathrm{X}$, then $F$ is closed since $\mathrm{X}$ is an L-closed space. In addtion, a subset $F$ is L-closed, hence it is P-subset and $\pi_{x \mid F \times Y}$ is closed where $\left(\pi_{x \mid F \times Y}\right)^{-1}(\mathrm{X})$ is Lindelöf [13]. 
Proposition 9. A space $(\mathrm{X}, \tau)$ is a P-space if and only if for each Lindelöf space $(Y, \sigma)$, the projection function $\pi_{x}: \mathrm{X} \times$ $\mathrm{Y} \rightarrow \mathrm{X}$ is closed.

Proof. $\Rightarrow)$ By Kuratowski's Theorem concerning compact topological spaces [16]. If a subset $F$ of $X$ is a closed and $\mathrm{X}$ is a P-space, if a point $y \notin \pi_{x}(F \times \mathrm{Y}), y \in V$ where $V$ is an open subset of $\mathrm{Y}$, then $(\mathrm{X} \times V) \cap F=\phi$ because $\mathrm{X} \times\{y\} \subseteq(\mathrm{X} \times \mathrm{Y}) \backslash F$ [1]. Hence, $\left.\pi_{x}(F \times \mathrm{Y})\right) \cap \mathrm{V}=\phi$, that is, a subset $\pi_{x}(F)$ of $X$ is closed [15].

Contrarily, each non-P-space is as follows, whenever $\pi_{x}$ is closed, a space $\mathrm{Y}$ has to be countably compact, thus $\mathrm{X}$ is non-P-space and this fact isobtained by Hanai, 1962.

Example 1. Consider the topological spaces $(\mathrm{X}, \tau)$ and $(\mathbb{R}, \sigma)$ where $\mathrm{X}$ is a $\mathrm{P}$-space and $\mathbb{R}$ is Lindelöf . The projection function $\pi_{x}: \mathrm{X} \times \mathbb{R} \rightarrow \mathrm{X}$ is not closed eventhough $\mathbb{R}$ is Lindelöf [10].

\section{Competing interests}

The authors declare that they have no competing interests.

\section{Authors' contributions}

All authors have contributed to all parts of the article. All authors read and approved the final manuscript.

\section{References}

[1] Al-labadi. M., Almuhur. E.,(2020), Planar of special idealization rings, WSEAS Transactions on Mathematics, 19(66), pp.606-609.

[2] Almuhur. E., Al-labadi. M., (2021), Pairwise Strongly Lindelöf, Pairwise Nearly, Almost and Weakly Lindelöf Bitopological Spaces, WSEAS Transactions on Mathematics, 20(16), pp.152-158.

[3] Engelking,(1977), General Topology, PWN-Polish Sci. Publ. Warszawa, Berlin.

[4] Ewert. J., (1986), On Pairwise Hausdorff Bitopological Spaces, Pedagogical University, Arciszewskiego. Poland, 22, pp.76-200.

[5] Fletcher. P., Hoyle. H.B., and Patty. C.W., (1969), The comparison of Topologies, Duke Mathematical Journal, 36, pp.325-331.

[6] Hdeib. H., and Pareek. C. M., (1986), On Spaces In Which Lindlof Sets are Closed, Q\&A in General Topology,4, pp.67-72.

[7] Hdeib. H., (1979), Contribution to the theory of [n,m]-compact, Paracompact and normal spaces, Ph.D. Thesis SUNY at Buffalo.

[8] Jankovic. D., Ellen. M., (1999), On Some Generalizations of L-closed spaces, Acta Mathematica Universitatis Comenianae, 2, pp.345-353.

[9] Kim. W., (1968), Pairwise Compactness, Publicationes Mathematicae Debrecen, 15, pp.87-90.

[10] Misra. A., (1972), Topological View of P-Spaces, General Topology and Its Applications, 2, pp.349-362.

[11] Milovančević. D., (1985), Property Between Compact and Strongly Countably Compact, Publications De L'Institut Mathema tique, 38, pp.193-201.

[12] Mukharjee. A., (2013), Some New Bitopological Notions, Publications De L'Institut Mathématique, 93,pp.165-172.

[13] Omer. N., Hdeib. H., Almuhur. E., Al-labad. M., (2021), ON P2-C-CLOSED SPACE IN BITOPOLOGICAL SPACE, Advances in Mathematics: Scientific Journal, 10(3), pp.1839-1843.

[14] Pahk. H., and Choi. D., (1971), Notes on pairwise compactness, Kyungpook Mathimatical Journal, 11, pp.45-52.

[15] Patty. W., (1967), Bitopological spaces, Duke Mathimaticla Journal, 34, pp.387-391.

[16] Weston. D., (1957), On the comparison of Topologies, Journal of London Mathimatical Society, 32, pp.342-354.

[17] Henriksen. M., Woods, (1988), Weak P-space and L-closed spaces, Q\&A in General Topology, 6, pp.201-206. 